\title{
The Role of SOD, Catalase, HSP-27, HSP-70, and TNF- $\alpha$ Expression in Apoptosis of Retinal Ganglion Cells After Intra Ocular Pressure Increase on Rattus Norvegicus
}

\author{
Nurwasis $^{1}$, Gatut Suhendro ${ }^{1}$, I Ketut Sudiana ${ }^{1}$ \\ ${ }^{1}$ Faculty of Medicine, Kampus A Universitas Airlangga Surabaya, St. Mayjen. Prof. Dr. Moestopo 47, \\ Surabaya Indonesia 60132
}

\begin{abstract}
The aim of this study is to investigate the involvement of Super Oxide Dismutase (SOD), Catalase, Heat Shock Protein 70 (HSP 70), Heat Shock Protein 27 (HSP 27) Retinal Ganglion Cells (RGCs) and Tumor Necrosis Factor $\alpha$ (TNF- $\alpha$ ) microglia in apoptosis RGCs and RGCs survival after transient periode of pressure induced ischemia/reperfussion injury of rat retina. The study design was randomized post test only control groups. Thirty one Sprague Dawley (SPD) rats were divided into 4 groups. 1 control group and 3 experimental groups. Experimental groups were induced by transient elevation of intraocular pressure (IOP) 110-130 $\mathrm{mmHg}$ for 45 minutes ( 7 rats), 60 minutes ( 7 rats), 75 minutes ( 7 rats). 28 rats were terminated at day 7 and 3 rats were terminated at first day for histology and immunohistochemistry staining examination. Result from the Expression of SOD, HSP 70 GSRs and TNF- $\alpha$ microglia were significantly different. $p=0,046$ and $\mathrm{B}=0.380$ for SOD; $\mathrm{p}=0.030$ and $\mathrm{B}=0,411$ for Hsp $70 ; \mathrm{p}=0.007$ and $\mathrm{B}=0,501$ for TNF- $\alpha$. Expression of catalase and HSP 27 GRCs were not significantly different. $p=0.203$ for catalase; $p=0.083$ for Hsp 27 . The increased expression of HSP 70 indicated strong correlation with increased level of apoptosis RGCs, $\mathrm{p}=0.046$ and $\mathrm{B}=0,473$. In conclusion, the result demonstrated that RGCs apoptosis survival in glaucoma correlates strongly with transient elevated IOP and is significantly associated with IOP induced changes in expression HSP 70 RGCs.
\end{abstract}

Keywords: Intraocular Pressure, IOP, HSP 70 RGCs, Apoptosis RGCs

\section{Introduction}

Glaucoma, a progresive optic neuropathy disease, is characterized by loss of retinal ganglion cell and their axons. This condition leads to irreversible visual loss. Death of RGCs in glaucoma human eyes and experimental animal models of glaucoma induced by apoptosis RGCs are still unclear. The elevated intraocular pressure was considered the prime factor for glaucoma ${ }^{1,2}$ Current theraphy that is directed at lowering IOP cannot completely stop the progression of the disease. Blindness caused by glaucoma is an issue in Indonesia and all over the world.

\section{Corresponding Author:}

I Ketut Sudiana

Faculty of Medicine, Kampus A Universitas Airlangga

Surabaya, St. Mayjen. Prof. Dr. Moestopo 47,

Surabaya Indonesia 60132

Email: i-ketut-s@fkp.unair.ac.id
Increased intra ocular pressure $110-130 \mathrm{mmHg}$ for 45 minutes, 60 minutes, and 75 minutes followed by reduction to normal TIO will cause reperfusion ischemia on the retina, in the area which contains cells of the retina and microglia ${ }^{3}$. Cells that undergo stress will promote changes in protein structure and function, so that the cells will be heading toward the death pathway or survival pathway. RGC which experience stress will produce ROS that is radical superoxide $(\mathrm{O} 2-)$, hydrogen peroxide $\left(\mathrm{H}_{2} \mathrm{O}_{2}\right)$ and hydroxyl radical $(\cdot \mathrm{OH})$. Increased ROS will be counterbalanced by an increase in endogenous anti oxidant namely superoxide dismutase (SOD) and catalase (CAT). RGCs which experience stress will also express Heat Shock Protein (HSP) 27 and 70. Microglias that undergo stress will express TNF- $\alpha^{4,5}$.

The purpose of this study is to explain the mechanism of RGCs apoptosis in glaucoma following reduction of IOP to normal level. 


\section{Methodology}

This study is a type of laboratory experimental research with randomized post-test only controls group design. The designs are as follows in Figure 1:

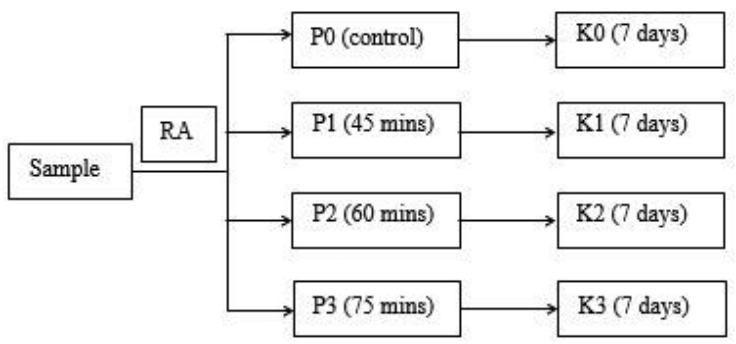

Figure 1: Research design approach

Where:

\section{RA: Random Allocation}

K0: Control group, without treatment an increase in IOP (P0), was sacrificed on day 7

K1: Treatment group with IOP increase for 45 minutes was then returned to normal IOP (P1), sacrificed on day 7

K2: Treatment group with IOP increase for 60 minutes was then returned to normal IOP (P2), sacrificed on day 7

K3: Treatment group with IOP increase for 75 minutes was then returned to normal IOP (P3), sacrificed on day 7

This research was conducted in a laboratory that is standard and has complete equipment and adequate experience in experimental animal maintenance, preparation of preparations as well as in immunohistochemical examination techniques under the guidance of doctoral quality consultants. For this reason, the research was carried out in a biochemical laboratory, immunohistochemical laboratory and electron microscope, Faculty of Medicine, Airlangga University. The time of the study was carried out for 6 months including the preparation, materials and tools, treatment, examination, data analysis and preparation of reports.

The sample of this study was male Sprague Dawley (SPD) type adult sex with a body weight of 250-300 grams, healthy condition and no abnormalities in the eye and general conditions.
After obtaining a homogeneous sample through screening with inclusion and exclusion criteria, a homogeneous sample group is divided by random allocation so that each sample member has the same opportunity to occupy its group.

In the process of carrying out the study, a dropout criterion was applied if the research subject experienced illness or death so that it could not fulfil the research procedure which took 7 days. Furthermore, rats were sacrificed and retinal tissue was collected around the optic nerve papillary to be made immunohistochemically preparations and staining. The collected data from histology and immunohistochemistry tests then carried out statistical analysis with the following details:

\section{Descriptive analysis of data}

2. Test for homogeneity

3. Multiple comparison test results of immunohistochemical examination (IHC) expression of SOD, Catalase, HSP70, HSP27, Apoptosis, Brn3b SGR and TNFa microglia.

4. Regression test to find out the causal relationship between the increase in intraocular pressure with variables SOD, Catalase, HSP70, HSP27, Apoptosis, Brn3b SGR and TNFa microglia.

\section{Results and Discussion}

Profile of Expression of Retinal Ganglion Cell Proteins and Microglia: Table 1 indicates that the standard deviation is 0.018 and the average SOD is 0.052 , so the coefficient of variation is 0.3 . Standard deviation of 0.015 and Catalase average of 0.036 , so that the coefficient of variation is 2.4. Standard deviation of 0.017 and mean HSP70 0.031 so that the coefficient of variation is 0.5 . Standard deviation of 0.011 and mean HSP27 0.041 so that the coefficient of variation is 0.3 . The standard deviation is 0.007 and the average TNF- $\alpha$ is 0.008 so the coefficient of variation is 0.9 . Standard deviation of 0.014 and mean apoptosis 0.008 so the coefficient of variation is 1.8. Standard deviation of 0.007 and Brn $3 b$ mean of 0.051 so that the coefficient of variation is 0.1 and there are homogeneous variables in the Brn3b variable.

Table 1: Mean and SD values for each variable in each group

\begin{tabular}{|c|c|c|c|c|c|c|c|}
\hline & SOD & Catalase & HSP70 & HSP27 & TNF- $\boldsymbol{\alpha}$ & Apoptosis & Brn3b \\
\hline Mean & 0.052 & 0.036 & 0.031 & 0.041 & 0.008 & 0.008 & 0.051 \\
\hline Standarddeviation (SD) & 0.018 & 0.015 & 0.017 & 0.011 & 0.007 & 0.014 & 0.007 \\
\hline Minimum & 0.039 & 0.020 & 0.021 & 0.028 & 0.000 & 0.000 & 0.044 \\
\hline Maximum & 0.072 & 0.051 & 0.051 & 0.048 & 0.012 & 0.024 & 0.057 \\
\hline
\end{tabular}


HSP70 expression in the treatment group was higher than the control group. HSP27 expression in the treatment group with duration of 45 minutes was higher than the control group, while the group duration of 60 and 75 minutes was lower than the control group. TNF $\alpha$ expression in the treatment group was higher than the control group. SGR apoptosis in the treatment group was higher than the control group. Living SGR in the treatment group with a duration of 45 minutes was higher than the control while the treatment group with duration of 60 and 75 minutes was lower than the control group.

\section{Immunohistochemistry Expression of Retinal} Ganglion Cell Proteins and Microglia: Figure 2 depicted the SOD1 Expression Retinal Ganglion cells with $400 \mathrm{X}$ magnification. This image shows an incision of retinal tissue with immunohistochemical staining. The red arrow shows a positive reaction to monoclonal antibodies (anti SOD1), while the green arrow does not react with monoclonal antibodies (anti SOD1). In this study there was an increase in SOD1 SGR expression, so as to neutralize the increase in free radicals, no oxidative stress and SGR apoptosis. While the expression of catalase SGR obtained a decrease in expression in the 75-minute group compared to the control group. These conditions indicate that catalase plays a major role in the detoxification of products from $\mathrm{SOD}$, namely $\mathrm{H} 2 \mathrm{O} 2$. This is consistent with the research of Goyal et al. ${ }^{6}$, there was an increase in levels of SOD and glutathione peroxidase in the serum of patients with open-angle glaucoma compared to cataract patients, whereas the levels of catalase did not differ. In another study conducted by Yuki et al. ${ }^{7}$, in experimental animals with SOD1 deficiency can result in SGR death. In other parts also found low SOD1 levels in the serum of normal pressure glaucoma sufferers compared to normal people.

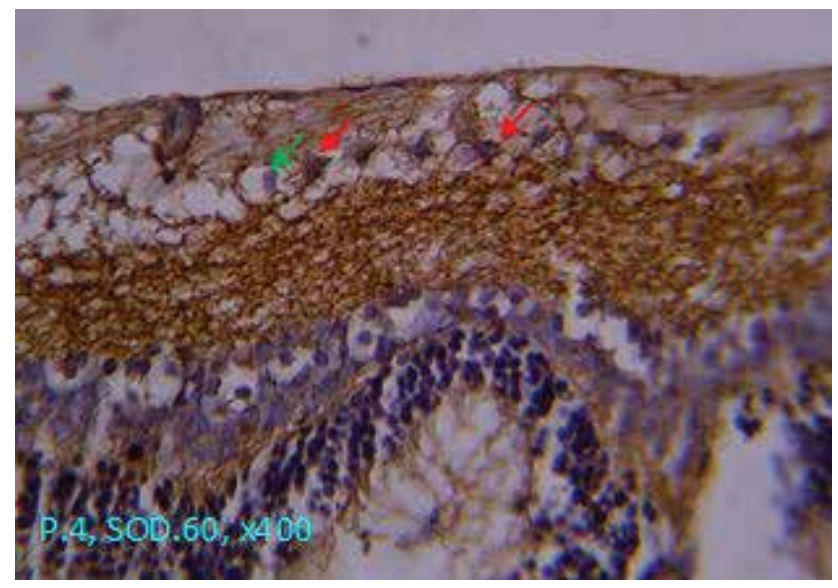

Figure 2: SOD Expression of Retinal Ganglion Cells at $400 \mathrm{X}$ magnification
Figure 3 presented the HSP expression 70 retinal Ganglion cells with 400X magnification. This image shows an incision of retinal tissue with immunohistochemical staining. The red arrow shows a positive reaction to monoclonal antibodies (anti HSP 70), while the green arrow does not react with monoclonal antibodies (anti HSP 70).Intracellular HSP 70 enhancement can act as anti-apoptosis by inhibiting the release of cytocrom $\mathrm{C}$ from mitochondria, HSP 70 can also inhibit the activation of AIF, APAF, and Caspase 3. Caspase 70 also plays an anti- apoptosis role at the pre mitochondrial level by binding to Bid and inhibiting P53. Intracellular HSP increases are followed by the increase of extracellular HSP which serves as an alarm for pro-inflammatory signals and immunomodulations such as IL 6 and TNF- $\alpha$. eHSP 70 also acts as microglia activation and stimulates phagocytosis ${ }^{8}$.

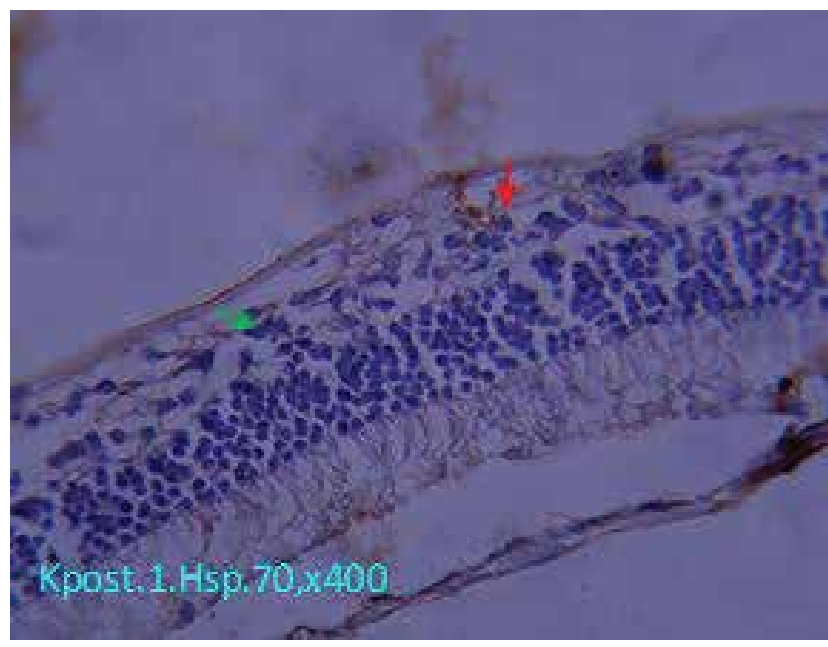

Figure 3: Expression of HSP 70 retinal Ganglion cells

HSP Expression 27 Retinal Ganglion cells with 400X magnification (refer Figure 4). This image shows an incision of retinal tissue with immunohistochemical staining. The red arrow shows a positive reaction to monoclonal antibodies (anti HSP 27), while the green arrow does not react with monoclonal antibodies (anti HSP 27).Schmitt et al. ${ }^{8}$, HSP 27 is a protein that functions to maintain cells from stressors and acts as an anti-apoptosis either through intrinsic or extrinsic pathways. Intracellular HSP 70 also acts as an antiaggregation, helping as transport, folding, and refolding as well as stabilizing filament and sito skeleton. On the other hand HSP 27 can activate the survival pathway through Phosphatidyl inositol 3 kinase (PI3-k). Akt/PKB and Nf-kB. Shields et al. ${ }^{9}$, hypothesized that HSP 27 acts as RAMPs, acting as immunomodulation and activating 
microglia membrane receptors to produce antiinflammatory cytokines such as IL-10. From the research data, it can be seen that with the increase in stressors following a decrease in HSP 27 SGR expression, Hsp 27 cannot play a role in SGR protection in both intracellular and extracellular.

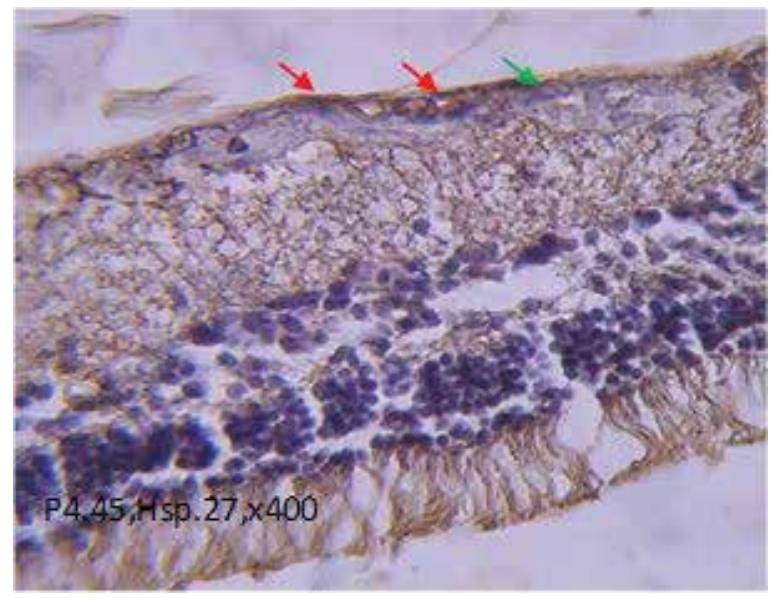

Figure 4: Expression of HSP 27 retinal Ganglion cells

Figure 5 shown the expression of TNF- $\alpha$ microglia with 400X magnification. This image shows an incision of retinal tissue with immunohistochemical staining. The red arrow shows a positive reaction to monoclonal antibodies (anti TNF- $\alpha$ ), while the green arrow does not react with monoclonal antibodies (anti TNF- $\alpha$ ).In the experimental animal model this study occurred similar to acute glaucoma in humans. At the biomolecular level of increase in TNF- $\alpha$ microglia there were differences in the duration of 75 minutes compared to the control group. TNF- $\alpha$ is known to have various functions as a proinflammatory cytokine, TNF- $\alpha$ can result in cell death through either the apoptosis or necroptosis process. SGR death at duration of 75 minutes may involve a process of necroptosis ${ }^{10,11}$.

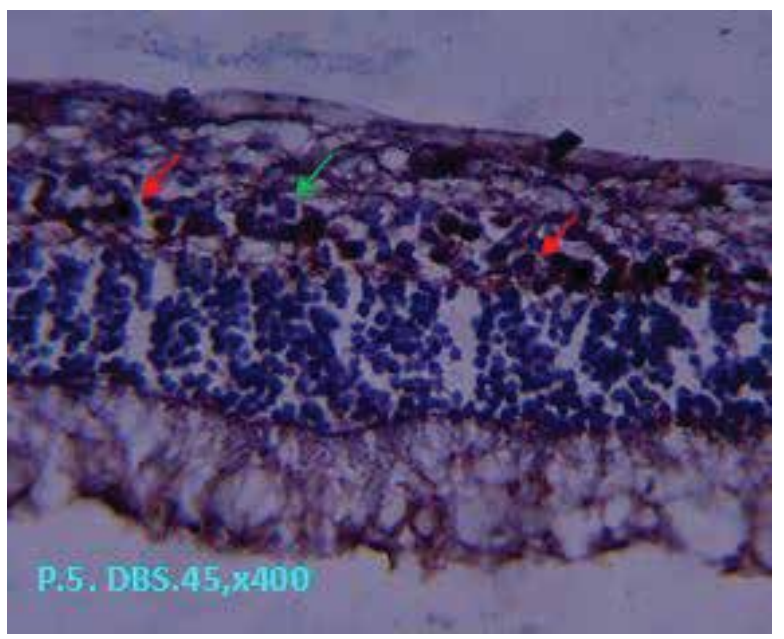

Figure 5: Expression of TNF- $\alpha$ microglia
ApoptosisexpressionretinalGanglioncellswith400X magnification is depicted in Figure 6. This picture shows an incision of retinal tissue with immunohistochemical staining using the TUNEL method. Red arrows indicate retinal ganglion cells undergoing apoptosis. Poon et al. ${ }^{11}$, in normal adult humans the process of apoptosis is very high, it is estimated that apoptosis of 1 million cells per second (physiological apoptosis) occurs. In this study, apoptosis occurred in the control group and increased in the treatment group. The increase in apoptosis occurred in the 60-minute duration group compared to the control group and the 45-minute treatment group, but there was no difference in SGR apoptosis in the 75-minute duration group compared to the control group.

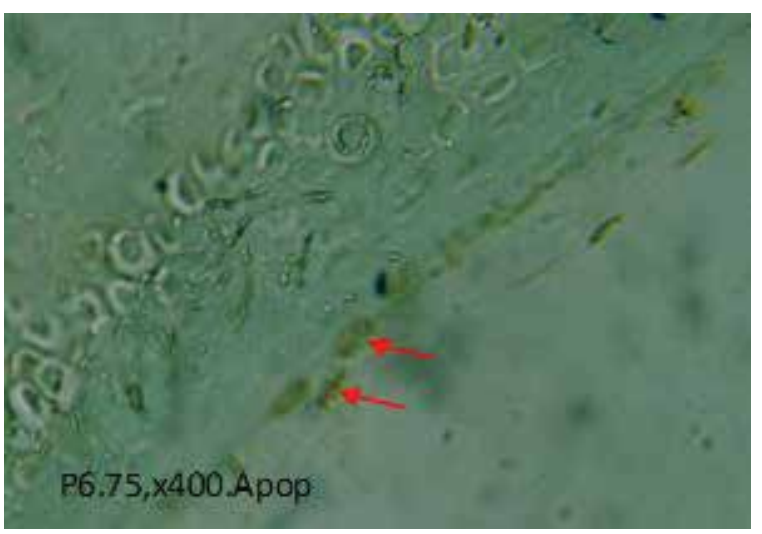

\section{Figure 6: Expression of Apoptosis in retinal Ganglion cells}

Figure 7 shown Brn3b expressions of retinal Ganglion cells with 400X magnification. This image shows an incision of retinal tissue with immunohistochemical staining. The red arrow shows a positive reaction to monoclonal antibodies (anti Brn3b), while the green arrow does not react with monoclonal antibodies (anti Brn3b). $\mathrm{Brn} 3 \mathrm{~b}$ is a marker used to identify SGR that survives. In this study, the lowest survival rate of SGR was 60 minutes compared to the control group (Figure 4.1). The survival decline in SGR occurred significantly at duration of 60 and 75 minutes compared to the control group.

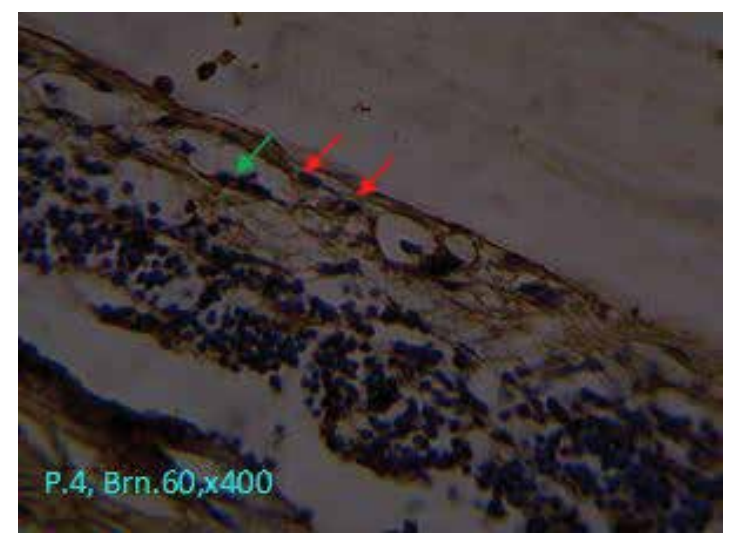

Figure 7: Expression of Brn3b retinal Ganglion cells 


\section{Conclusions}

In conclusions, after the increase of IOP followed by decrease of IOP to normal level will result in increased expression of SOD, HSP70 RGC and TNF- $\alpha$ RGCs microglia. HSP 70 is the main apoptotic RGC biomarkers.

Ethical Clearance: Taken from the committee

Source of Funding: Nil

Conflict of Interest: Nil

\section{References}

1. S. Gupta, P. Agarwal, R. Saxena, S. Agrawal, R. Agarwal. Indian Journal of Ophthalmology.2009;57(257).

2. R.R. Allingham, M.B. Shields. Shields Textbook of Glaucoma (Wolters Kluwer/Lippincott Williams \& Wilkins Health, Philadelphia, PA). 2011.

3. J.M.K. Kwong, J. Caprioli, Neuromethods Animal Models for Retinal Diseases. 2009;191.
4. M. Karlstetter. Innate Immunity and the Eye. 2013;12.

5. A. Sierra, M.-È. Tremblay. Microglia in Health and Disease.2014;3.

6. A. Goyal, A. Srivastava, R. Sihota, J. Kaur. Current Eye Research. 2014;39(823).

7. K.Yuki, T. Yoshida, S. Miyake, K. Tsubota, Y. Ozawa. Experimental Eye Research. $2013 ; 115(230)$.

8. E. Schmitt, M. Gehrmann, M. Brunet, G. Multhoff, C. Garrido. Journal of Leukocyte Biology. 2006;81(15).

9. A.M. Shields, G.S. Panayi, V.M. Corrigall. Clinical \& Experimental Immunology. 2011;165(292).

10. J.C. Reed, D.R. Green. Apoptosis: Physiology and Pathology (Cambridge University Press, New York).2011.

11. I.K.H. Poon, C.D. Lucas, A.G. Rossi, K.S. Ravichandran. Nature Reviews Immunology. 2014;14(166) 